\title{
Clipboard
}

\section{A gene involved in nematode feeding behaviour}

Recent studies on feeding behaviour in the soil nematode Caenorhabditis elegans provide a striking illustration of how genetic and environmental influences together mould complex behaviour.

C. elegans worms inhabit decaying organic matter in the soil and feed on different species of bacteria. Cassada first noted in 1987 that in the presence of food, wild strains of C. elegans exhibit one of two distinct feeding behavioural patterns, known as 'solitary' and 'social'. Since then, this has been confirmed in the case of every one of 17 natural isolates from all over the world (de Bono and Bargmann 1998). Solitary feeders forage alone on a bacterial lawn (figure 1A). The common laboratory strains N2 (originally isolated from England) and TR403 (from Wisconsin, USA) belong to this category. In contrast, social feeders tend to clump with each other while feeding (figure 1B). Some isolates from Hawaii, Australia, Germany and England show such social feeding. In one instance, social and solitary wild strains were isolated from the same sod of soil (de Bono and Bargmann 1998). It must be emphasized that the two classes of isolates differ in their feeding behaviour only under conditions of a plentiful supply of food bacteria, underlining the importance of the environment in determination of this behaviour. Also, the aggregation behaviour is enhanced by an increased density of worms (de Bono et al 2002).

A clue that the behavioural difference between solitary and social feeders might be correlated with variation at a single genetic locus came with the isolation of three mutants of the solitary strain N2 that showed social feeding (de Bono and Bargmann 1998). The three point mutations were all recessive to the wild-type and did not complement each other. This indicated that they shared the same locus. The mutations were mapped to a single gene on the X-chromosome, named npr-l (for neuropeptide receptor resemblance; see below). In other experiments, back-crossing was carried out for 10 generations between the naturally occurring social strain RC301 from Germany and N2. The progeny in every cross were selected for a marker close to the $n p r-1$ locus. The DNA of the quasi-inbred strains thus produced contained sequences from the social strain around the npr-1 locus but had N2 sequences elsewhere. All the independently outcrossed strains exhibited strong social behaviour similar to the parental RC301 strain. Once again, it appeared that allelic variation in single region of the genome of the social strain, perhaps in just npr-1, was sufficient to change the behavioural phenotype (de Bono and Bargmann 1998).

NPR-1 is thought to encode a 7-transmembrane G-protein-coupled receptor, similar to members of the neuropeptide $\mathrm{Y}$ receptor family. With the exception of just one base position, the $n p r-1$ coding region of $1.4 \mathrm{~kb}$ was found to be identical in all 17 natural isolates tested. But the variation at that position

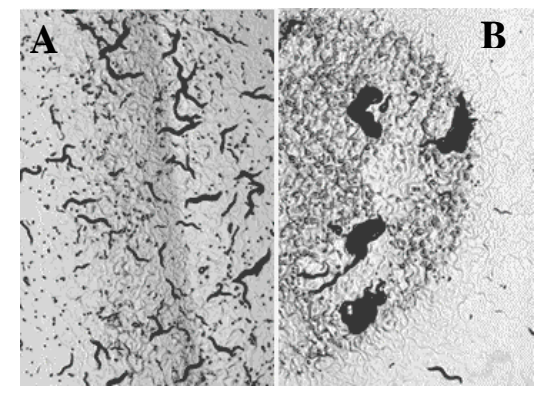

Figure 1. The solitary and social strains of C. elegans behave differently in the presence of bacteria. (A) Solitary worms disperse evenly across a bacterial lawn and browse alone. (B) Social animals aggregate together into clumps (de Bono and Bargmann 1998; courtesy, M de Bono). 
was critical. The twelve social feeding strains had a TTT codon [implying that phenylalanine (F) was amino-acid number 215 in the predicted NPR-1 sequence] in the same position at which the remaining five solitary strains had a GTT codon [implying that in them amino-acid number 215 was valine (V); de Bono and Bargmann 1998]. Thus despite their geographically distinct origins, the wild isolates displayed a perfect correlation between the 215th residue of NPR-1 and feeding behaviour, at least in the sample of worms tested. A comparison of the NPR-1 sequence with that of other G-proteincoupled receptors indicated that residue 215 was probably located in the third intracellular loop of the receptor. This loop is the site of protein-protein interaction and any change in sequence of this loop might alter $\mathrm{G}$ protein specificity and/or affinity and hence affect downstream signalling. The 'solitary' $215 \mathrm{~V}$ allele is dominant over the 'social' $215 \mathrm{~F}$ allele. Accordingly, expression of $n p r-1215 \mathrm{~V}$ from a transgene was sufficient to make a $n p r-1215 \mathrm{~F}$ worm behave like its solitary counterpart. But $n p r-1$ null mutants are hypersocial, implying that both the natural variants depress social feeding behaviour upon activation (de Bono and Bargmann 1998). npr-1 215V worms might be solitary because this isoform inhibits social feeding more potently than the $215 \mathrm{~F}$ isoform.

Behavioural differences in populations can rarely be accounted for by variation at a single locus, and these studies would seem to belong to the rare class. However, it turns out that one should be cautious in coming to such a conclusion.

The simple nervous system of the $C$. elegans provides opportunities to investigate the role of nerve cells and their connections in fostering solitary or social feeding. An adult worm, about $1 \mathrm{~mm}$ long, contains 302 neurons. Each of these neurons can be uniquely identified by morphology and anatomical position. The first study investigated how and where NPR-1 acts. When a green fluorescent protein (GFP)-tagged version of the $n p r-1215 \mathrm{~V}$ allele was expressed under its native promoter in $n p r-1$ null (therefore hypersocial) worms, a number of neurons in the transgenic worms showed fluorescence - and, as mentioned earlier, the worms themselves displayed solitary feeding. By using different promoters to drive expression of the transgene in various subsets of neurons, it was inferred that expression in just three pairs of sensory neurons was sufficient to suppress social feeding (Coates and de Bono 2002). These three pairs of neurons, AQR, PQR and URX, are directly exposed to the pseusocoelomic body fluid. This opens the possibility that distant neurons in the body of the worm can regulate the activity of AQR, PQR and URX by secreting neuropeptides or neuromodulators in the body fluid - an observation that parallels the well-known fact that humoral signals regulate mammalian feeding.

The knowledge that both food and population density influence feeding behaviour helped in the design of another experimental strategy. A collection of genetically defined mutants that were impaired in the reception of various environmental stimuli like attractants, repellants and touch was screened for feeding behaviour. This led to the identification of four genes which, when mutated, also showed altered feeding behaviour (de Bono et al 2002). Two code for cation channel subunits in chemosensory neurons and are involved in avoidance behaviour to noxious stimuli such as benzaldehyde and 1-octanol. The other two genes encode factors required to localize chemosensory receptors to sensory cilia. Laser ablation of the nociceptive neurons ASH and ADL abolished social feeding behaviour in $n p r-1$ mutant worms. Streptomycin-killed Escherichia coli did not induce social feeding; but exposure to volatile substances from live E. coli cells did (de Bono et al 2002). Thus, bacteria seem to produce compounds detected by nociceptive neurons that stimulate social feeding and related behaviours. This led to the suggestion that aggregation during feeding might have evolved as a stress response to aversive stimuli produced by bacteria. It appears odd to think of food as a source of an aversive stimulus. On the other hand, E. coli and many soil bacteria that form part of the diet of C. elegans in nature can kill worms under certain conditions such as presence of certain virulence factors in bacteria, the age of the worm host and so on.

Recently, using a candidate-ligand approach, FMRFamide related neuropeptides (FaRPs) were identified as NPR-1 ligands (Rogers et al 2003). These neuropeptides (encoded by flp18 and flp21) have the amino acid sequence of FMRF at their carboxy-terminus with an amide modification on the C-terminal Phe residue. Loss of function studies and overexpression of genes encoding these peptides showed that the peptides can influence feeding behaviour. Injection of chemically synthesized peptide in wild social worms strongly suppressed aggregation behaviour, confirming that NPR-1 ligands inhibit social feeding. The genetic and neurobiological data can be integrated to propose a hypothetical and preliminary circuit that underlies feeding behaviour (figure 2). 


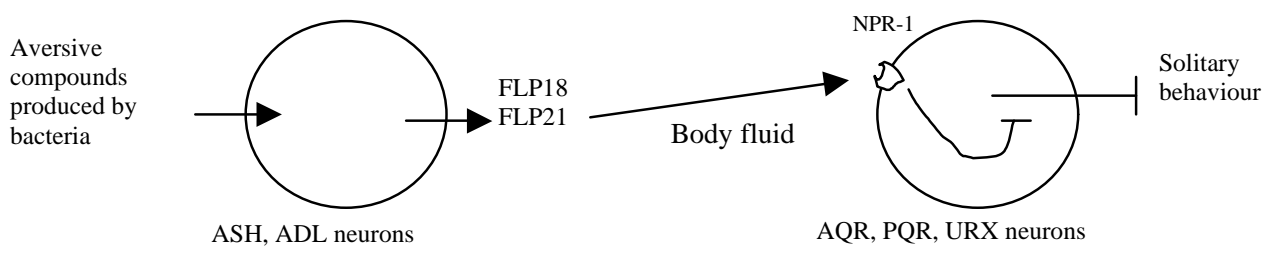

Figure 2. Schematic of a possible NPR-1 neural circuit: Bacteria emit an aversive odour that stimulates ASH and ADL neurons to secrete FLP-18, FLP-21 neuropeptides in the body fluid. AQR, PQR and URX neurons act to suppress solitary behaviour, promoting social feeding. These also express NPR-1, which binds FLP-18, FLP-21 in the body fluid. This activates NPR-1, which results in inhibition of neuronal activity of AQR, PQR and URX. NPR-1 $215 \mathrm{~V}$ isoform is more potent in inhibiting the neurons than $215 \mathrm{~F}$ isoform. Thus neurons with $215 \mathrm{~V}$ isoform fail to inhibit solitary feeding. Consequently, this isoform promotes solitary behaviour while the other isoform promotes social feeding (see text for more details and references).

What are the selective forces that affect feeding behaviour? Which (if any) of the two feeding patterns is more advantageous? The solitary and social feeders come from many locations, and in one case both were isolated from the same site. The two may represent alternative foraging strategies that are favoured under different - and as yet unknown - environmental conditions. In a given environment one strategy could be superior to the other. Alternatively, they may represent examples of two behavioural phenotypes that confer equal fitnesses when averaged over the spatial range of the populations or over the life cycle or both. In that case, they would constitute an interesting example of a genetic polymorphism underlying an evolutionary stable strategy (Gadgil et al 1982).

A comparison of $n p r-1$ sequences in different Caenorhabditis species shows that the $215 \mathrm{~F}$ allele is widespread among closely related species (Rogers et al 2003). As mentioned earlier, the $215 \mathrm{~V}$ allele is present only in a subset of $C$. elegans wild isolates. This points to the 'social' allele being more ancient than the 'solitary' one. Does this also mean that social behaviour is ancestral? It turns out that even when they carry the $215 \mathrm{~F}$ allele, individuals from other Caenorhabditis species are solitary or weakly social feeders ( $\mathrm{M}$ de Bono, personal communication). Therefore the correlation between the allelic state at the npr-1 locus and feeding behaviour does not hold in other species. This means that other genomic loci (as already evident in the case of $C$. elegans) and the environment are important in shaping feeding behaviour.

Even though many questions remain, it is clear that $C$. elegans has much to offer in understanding how genes, neurons, circuits and the environment guide animal behaviour.

\section{Acknowledgements}

I thank Mario de Bono for critical comments on an earlier draft.

\section{References}

de Bono M and Bargmann C 1998 Natural variation in a neuropeptide V receptor homolog modifies social behaviour and food response in C. elegans; Cell 94 679-689

de Bono M, Tobin D, Davis W, Avery L and Bargmann C 2002 Social feeding in Caenorhabditis elegans is induced by neurons that detect aversive stimuli; Nature (London) 419 899-903

Coates J and de Bono M 2002 Antagonostic pathways in neurons exposed to body fluid regulate social feeding in Caenorhabditis elegans; Nature (London) 419 925-929

Gadgil S, Nanjundiah V and Gadgil M 1980 On evolutionarily stable compositions of populations of interacting genotypes; J. Theor. Biol. 84 737-759

Rogers C, Reale V, Kim K, Chatwin H, Li C, Evans P and de Bono M 2003 Inhibition of Caenorhabditis elegans social feeding by FMRFamide-related peptide activation of NPR-1; Nat. Neurosci. 6 1178-1185

RITWICK SAWARKAR

Department of Molecular Reproduction,

Development and Genetics,

Indian Institute of Science,

Bangalore 560 012, India

(Email,ritwick@mrdg.iisc.ernet.in)

J. Biosci. | Vol. 29 | No. 1 | March 2004 